\title{
Study on Agricultural Condition Monitoring and Diagnosing of Integrated Platform Based on the Internet of Things
}

\author{
Jinying $\mathrm{Yu}$ and Wei Zhang \\ Information Institute of Science and Technology, Beijing Academy \\ of Agriculture and Forestry Sciences, Beijing 100097, China
}

\begin{abstract}
In order to meet the needs of modern agriculture, with the internet of things and related technologies, the author has developed a set of agricultural condition monitoring and diagnosing of integrated platform for experiment. The platform used the client and server mode, include environmental factor of monitoring platform, intelligent decision sub platform and expert remote science and technology advisory services subsidiary platform. Environmental factors collected by data acquisition module, and transmit through network. Audio and video data used the XMPP protocol extension Jingle transmission; Use the knowledge base, fuzzy theory, self learning algorithm of intelligent to achieve decision-making and control. The platform has been experimented in the vegetable greenhouses, which can carry out remote supervisory and automated control and expert remote diagnosis and consultation, improving the facilities agriculture management level.
\end{abstract}

Keywords: Internet of Things, Agricultural condition monitoring, Automatic regulation, Distance-vision consulting and diagnosing.

\section{Introduction}

With the development of information technology, more and more intelligent systems have been applied to agricultural production. The strawberry grower Norcal Harvesting living in Oxnard of California the United States, installed a set of networking system developed by Climate Minder. According to the air and the soil condition, the system can real-time track plant condition. It can automatically trigger the related behaviors, such as water or adjust the temperature [1]. The system based on GPRS wireless communication and the internet technology has been developed in the Jiangsu University, which can remotely collect and monitor the environment factor data in the aquatic products breeding[2]. Zhengyi Ren and Juanjuan Pan constructed a system that can remote diagnostics and monitoring the facilities gardening pests [3]. The Information Institute of Science and Technology Beijing Academy of agriculture and forestry sciences developed a remote video consultation and diagnosis system [4]. But all of the above systems are not forming an organic whole. The agricultural condition monitoring and diagnosing of integrated platform 
based on the internet of things can organically integrate the remote monitoring the produce environmental factor, the video surveillance of production site, automatic control and remote video consultation for the diagnosis all together, which can improve the agricultural fine management level.

\section{Platform Construction}

The agricultural condition monitoring and diagnosing of integrated platform based on the internet of things includes environmental factor of monitoring platform, intelligent decision sub platform and expert remote science and technology advisory services subsidiary platform. It is mainly composed of a sensor, camera, internet, intelligent decision and other network technology and device.

\subsection{The Environmental Factor of Monitoring Platform}

The environmental factor of monitoring platform is mainly composed of a sensor, camera, switches and other components. Various sensors are responsible for collecting each environmental factor data, transmission to the server by the network. The data collected by the camera transmit to the concentrator. All the real time environment factor data and the video data concentrate through the switch, then transmit the data to the server by the network. The platform used the cable and the switch completes the production monitoring of the temperature and humidity, illumination, carbon dioxide and other environmental factors and the video monitoring. The installation is simple, and has the strong stability and the strong data exchange [5-7].

\subsection{The Intelligent Decision Sub Platform}

The intelligent decision sub platform comprises input module, output module, threshold module, decision module, alarm control module. All kinds of monitoring data input through the input module. When the data reaches the threshold, the platform will generate an alarm signal, sound, Email, SMS and other forms of realtime alarm, for reminding the user to accurately grasp the watering, shading and other in the appropriate time; According to the abnormal situation, decision module can also independently find solutions from the knowledge base, then the platform transfers the control information through the output module to each actuator, automatically control temperature, humidity etc.with right measures.

\subsection{The Expert Remote Science and Technology Advisory Services Subsidiary Platform}

The expert remote science and technology advisory services subsidiary platform is mainly composed of the video module, audio module, text communication module, application sharing module, file transmission module, communicated in XMPP 
protocol. The video module carries out video compression, transmission and decompression. The audio module provides the voice compression, transmission and decompression, and the video module combines with the audio module, both of the modules can realize the remote "face to face" real time communication between the experts and the users for remote diagnosis. When the users and experts need to communicate by text, the text communication module will come into play. Through the application sharing module the experts can reveal some applications to the user, such as PPT, word, furtherly they can transmit information to the users through the file transmission module. According to the need, the platform also can carry out remote training.

\section{The Structure and Principle of Platform}

\subsection{The structure of platform}

The structure of platform is divided into two parts, server and client. The server locates in the center, which is responsible for the exchange of information and information management. The clients are divided into two genres: one genre is the information collection client, including the sensors of temperature, humidity, light and carbon dioxide and other environmental factors, camera as well as intelligent decision platform. The other genre is the user client, including the internet monitoring advisory terminal and the mobile phone monitoring advisory terminal [8,9]. In the course of practical application, the environmental factor of monitoring platform continuously collects data, when the data is abnormal, the intelligent decision sub platform will automatically alarm and control; and the users can view the environment factor of real-time data, history data and video scene through the client; when the users meet with the complex problems, they can remotely contact with expert "face to face".

\subsection{The Principle of Platform}

The server-side realize environmental factor data acquisition and management, video data collection and management, expert "face to face" information communication and management with the XMPP protocol, Socket technology, combination authentication. The environmental factor data can be operated with the smart sensor, RJ45 modules, cable, Socket technology, network camera, etc. With H.264 media encoding technology, G.723.1, AMR coding technology, XMPP protocol the platform can realize remotely expert "face to face" consulting diagnosis. The intelligent decision sub platform using the knowledge base, fuzzy theory, self learning algorithm to decision control. The multi point control unit through the XML language realizes each module message control. The user client using H.264 media encoding technology, G.723.1, Socket technology, XMPP protocol, combination authentication technology, implements consulting diagnosis, video monitoring, environment monitoring and other functions. Technical diagram as shown below. 


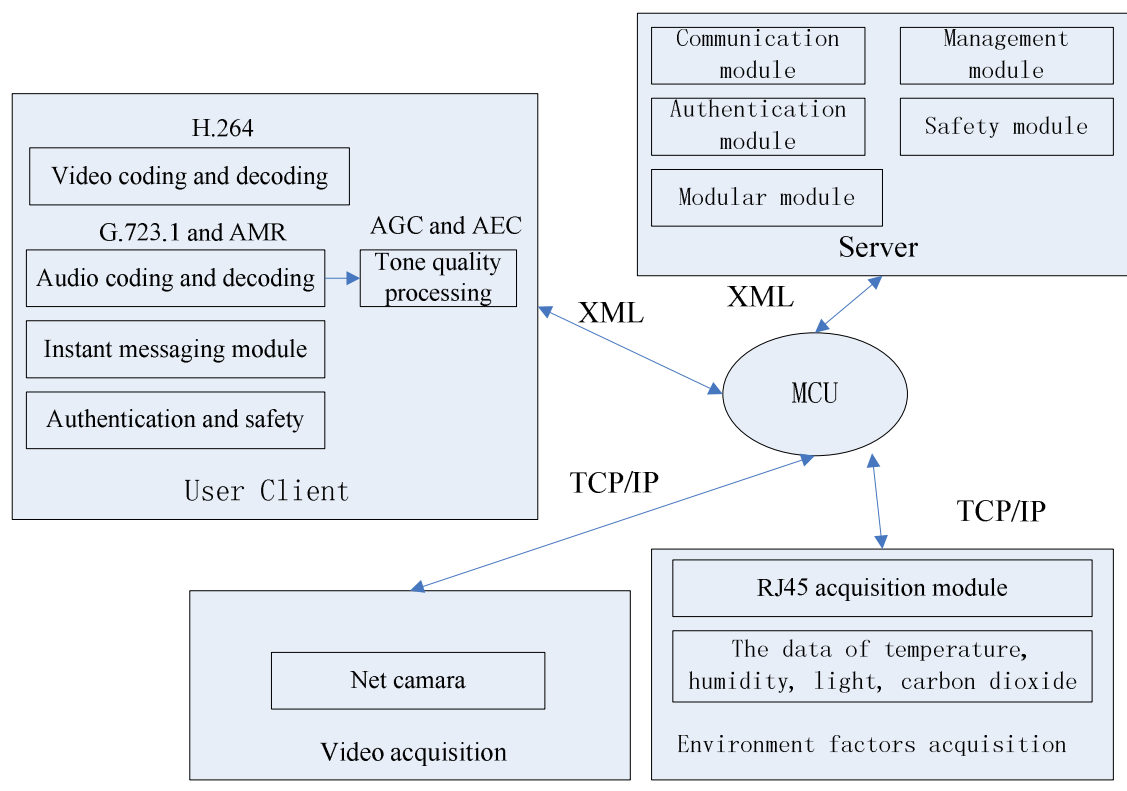

Fig. 1. System structure diagram

\section{$3 \quad$ Platform Test and Analysis}

\subsection{Experimental Design}

The vegetable greenhouse cultivation of cucumber has been designed as an experimental environment and the author has completed the crop condition monitoring diagnosis experiment [10-12], in the experiment, the author has designed four sensors such as Temperature, humidity, light, carbon dioxide ,etc. All the data are collected through the RJ45 collection module and they are transmitted to the server through the network. The users can monitor the data through the Web; the author has designed the threshold for each factor, when the monitoring value exceeds the threshold, it will alarm and tell us which environmental factor is unnormal in the greenhouse. The platform has been placed on the internet; The experts can face to face communicate with the users and guide the users in actual production through the XMPP server.

\subsection{Experimental Materials}

Four sensors: the temperature sensor, the humidity sensor, the light sensor, the carbon dioxide sensor (supply voltage of $12 \mathrm{~V} \mathrm{DC}$, output 4-20mA current signal); RJ45 acquisition module (8 inputs, the network output); the Server; Framework 3.5; the PC computer terminal with USB camera; the heating equipment: hot stove. 


\subsection{Experimental Method}

The experimental utilize the field tests. In the $70 \mathrm{~m} \times 10 \mathrm{~m}$ vegetable greenhouses, the experimenters have installed one temperature sensor, one humidity sensor, one light sensor, one carbon dioxide sensor in the center. The experimenters have erected one network camera at the end of the greenhouse. In order to complete the experiment, the experimenters in the greenhouse will man-made change some environmental factor specifically, such as use the hot stove to enhance the greenhouse temperature to check whether there is an alarm. Experts can remote access check local actual condition by the PC.The local experimenters also through the local computer terminals check the experts' calls.

\subsection{Experimental Results}

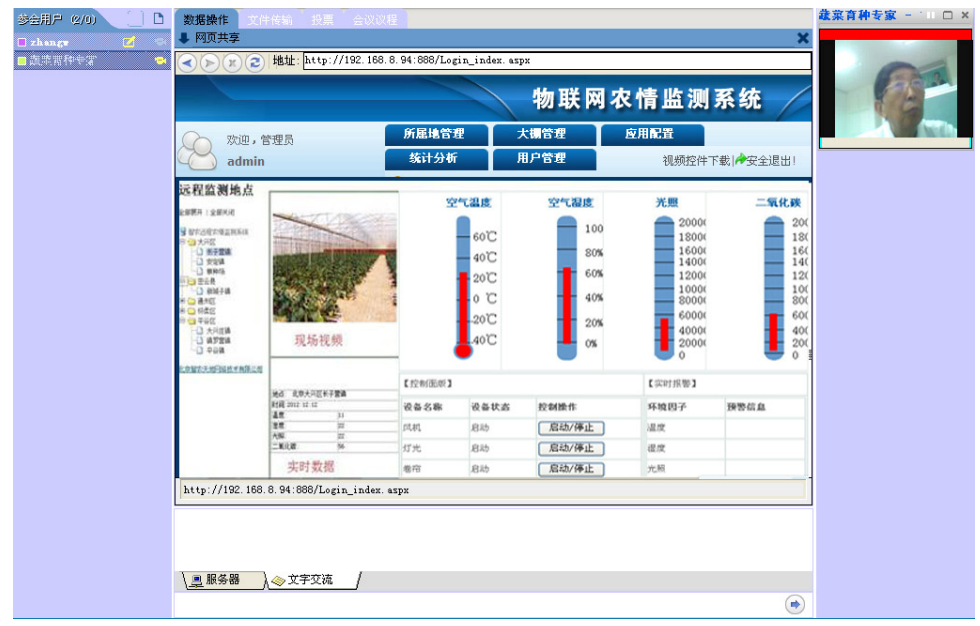

Fig. 2. The experimental results

In the Experiment the various functions of the system platform have been verified. The expert can real-time monitor the vegetable greenhouse through the browser, he can read each environmental factor, such as data, video etc., check the local alarm information of the high temperature, remotely "face to face" exchange successfully with local experimenters. The experimental result show as above Fig.2, the expert is viewing the environmental factors data.

\section{Conclusion and Discussion}

The agricultural condition monitoring and diagnosis of integrated platform based on the internet of things creatively put environmental factor remote monitoring, remote monitoring of production site, production automation control and remote video 
consultation for the diagnosis organically together. All the data of environmental factors and the live video are collected through the network cable. They can transfer to the server side by the switch. The intelligent decision sub platform on the server can compare the environmental factors data with the threshold all the time. When the monitoring value exceeds the threshold, the system will alarm, and the sub platform will make an order to the actuator to open or close, to realize automatic regulation; the users who have known the alarm can manual control by themselves. The users can remotely view site status at any time. When the plant diseases and there are insect pests, after logining the experts can not only see crop diseases, they also can view real-time and historical data, which is significant to improve the accuracy of diagnosis to plant diseases and insect pests, timely to resolve the disease, to reduce the loss .

Along with the internet technology development, the platform function need to be extended to further standardize; how to further standardize networking standards, how to transplant $3 \mathrm{G}$ mobile network platform to realize mobile monitoring and consulting diagnosis etc, all these need to continue to study and explore [13-16].

\section{References}

1. Jin, P.: With the internet of things to promote the modern facility agriculture. Agricultural Machinery Market 3, 27-28 (2010)

2. Fan, Q., Lai, R., Qiu, Q., Chen, Y., Lin, T., Zhang, X.: Development and application of online distant diagnosis of tobacco diseases and insects. Chinese Tobacco Science 29(1), 60-61 (2008)

3. Ren, Z., Pan, J., Gu, P., Yang, J., Su, N., Ren, A., et al.: Establishment of diagnosis and remote monitoring system for diseases and pests in protected cultivation. Acta Agriculturae Boreali-Occidentalis Sinica 19(3), 62-65 (2010)

4. Zhang, W., Yu, J., Yu, F., Luan, R.: Study on agricultural distance monitoring and diagnosing integration platform based on XMPP. Chinese Agricultural Science Bulletin 27(11), 151-154 (2011)

5. Wang, Y., Bai, Z.: Design of system auto controlling and measurement of parameters of environment formodern greenhouse. Microcomputer Information 24(20), 140-141 (2008)

6. Ding, X., Jin, H., Zhang, H., Yu, J.: Changes of illuminations and light transmissivities in different greenhouses. China Cucurbits and Vegetables 24(1), 1-4 (2011)

7. Zhang, F., Bian, X.: Middle ware technology of internet is the important links in internet of things chain. Sci-tech Innovation and Productivity 3, 41-43 (2011)

8. Wu, H.: The enter of $3 \mathrm{G}$ applications into the era of the internet of things. Journal of Changchun Normal University 30(1), 66-68 (2011)

9. Wang, D., Nie, Y., Wang, L., Li, C.: Research on strategic conception of green agricultural products application platform of internet ofthings in Heilongjiang reclamation Area. Ecological Economy 233(12), 145-147 (2010)

10. Jin, P.: The internet of things application in the modern facility agriculture. Agriculture Engineering Technology (Agricultural Product Processing Industry) 5, 40-41 (2010)

11. Jin, P.: The internet of things and the modern facility agriculture. Agriculture Machinery Technology Extension 2, 45 (2010) 
12. Lai, W.: Agricultural internet of things application in the modern flower industry. China Flowers \& Horticulture 20, 28-29 (2010)

13. Sun, Z., Du, K., Yin, S.: Development trend of internet of things and perspective of its application in agriculture. Agriculture Network Information 5, 5-8 (2010)

14. Cao, J., Fan, Y., Zhu, K., Wang, Z., Dai, Q.: Analysis of internet of things in Jiangsu agricultural development. Jiangsu Journal of Agricultural Sciences 26(6), 1402-1405 (2010)

15. Zhao, J., Wang, Y., Yang, M., Tan, X.: IOT application in agricultural pest disasters. Communications Technology 43(11), 49-51 (2010)

16. Ma, X., Wang, W., Han, J., Yuan, S., Li, P., Pang, C., et al.: To accelerate the realization of agricultural modernization with internet of things technology. Journal of Shanxi Agricultural Sciences 39(4), 376-378 (2011) 